\title{
A Kato Type Theorem on Zero Viscosity Limit of Navier-Stokes Flows
}

\author{
Xiaoming Wang* \\ Department of Mathematics \\ Iowa State University \\ Ames, IA 50011 \\ U.S.A.
}

Dedicated to Professors Ciprian Foias and Roger Temam with great admiration and friendship on the occasion of the retirement of Professor Foias and on the occasion of the sixtieth birthday of Professor Temam

\begin{abstract}
We present a necessary and sufficient condition for the convergence of solutions of the incompressible Navier-Stokes equations to that of the Euler equations at vanishing viscosity. Roughly speaking convergence is true in the energy space if and only if the energy dissipation rate of the viscous flows due to the tangential derivatives of the velocity in a thick enough boundary layer, a small quantity in classical boundary layer theory, approaches zero at vanishing viscosity. This improves a previous result of T. Kato (1984) in the sense that we require tangential derivatives only while the total gradient is needed in Kato's work. However we require a slightly thicker boundary layer. We also improve our previous result where only sufficient conditions were obtained. Moreover we treat more general boundary condition which includes Taylor-Couette type flows. Several applications are presented as well.
\end{abstract}

*wang@math.iastate.edu, 1-(515)294-1752 (T), 1-(515)294-5454(F) 
keywords: Zero viscosity limit, Navier-Stokes equations, Euler equations, energy dissipation rate, boundary layer

\section{Introduction}

One of the fundamental system governing the motion of fluids like air and water under normal conditions is the Navier-Stokes equations for incompressible homogeneous Newtonian fluids:

$$
\begin{aligned}
\frac{\partial \mathbf{u}^{\nu}}{\partial t}+\left(\mathbf{u}^{\nu} \cdot \nabla\right) \mathbf{u}^{\nu}-\nu \Delta \mathbf{u}^{\nu}+\nabla p^{\nu} & =\mathbf{f}, \text { in } \Omega, \\
\operatorname{div} \mathbf{u}^{\nu} & =0 \text { in } \Omega, \\
\mathbf{u}^{\nu} & =\mathbf{b} \text { on } \Gamma, \\
\mathbf{u}^{\nu} & =\mathbf{u}_{0} \text { at } t=0,
\end{aligned}
$$

where $\mathbf{u}^{\nu}=\left(u_{1}^{\nu}, u_{2}^{\nu}, u_{3}^{\nu}\right)$ is the velocity field in the Eulerian coordinates, $p^{\nu}$ is the pressure, and $\mathbf{f}=\left(f_{1}, f_{2}, f_{3}\right)$ is the external body force, the positive constant $\nu$ is the kinematic viscosity. The velocity $\mathbf{b}$ at the boundary satisfies

$$
\mathbf{b} \cdot \mathbf{n}=0 \text {, }
$$

where $\mathbf{n}$ is the unit outward normal to $\Gamma=\partial \Omega$. This means that the boundary is impermeable. This includes the case of Taylor-Couette type flows among others. The boundary condition sometimes is referred as characteristic boundary condition since the boundary consists of stream lines all the time.

The interested reader may consult the books of Constantin and Foias (1988), Ladyzhenskaya (1969) or Temam (1984) for the mathematical theories of the Navier-Stokes equations.

For realistic fluids like air and water, the kinematic viscosity is very small and hence we may formally set it to zero and arrive at the Euler system for incompressible inviscid (dry) fluids:

$$
\begin{aligned}
\frac{\partial \mathbf{u}^{0}}{\partial t}+\left(\mathbf{u}^{0} \cdot \nabla\right) \mathbf{u}^{0}+\nabla p^{0} & =\mathbf{f}, \text { in } \Omega, \\
\operatorname{div} \mathbf{u}^{0} & =0 \text { in } \Omega, \\
\mathbf{u}^{0} \cdot \mathbf{n} & =0 \text { on } \Gamma, \\
\mathbf{u}^{0} & =\mathbf{u}_{0} \text { at } t=0 .
\end{aligned}
$$


The natural question is then whether such an approximation can be justified via the zero viscosity limit of the Navier-Stokes equations.

The purpose of this article is to give a necessary and sufficient condition that the solutions of the Navier-Stokes equations (1) converge to that of the Euler equations (6) in the energy space $\left(L^{2}(\Omega)\right)$ at vanishing viscosity $(\nu \rightarrow 0)$.

It is clear that due to the disparity of boundary conditions between the viscous problem (1) where the whole velocity is specified at the boundary and the inviscid problem (6) where only the normal velocity is specified at the boundary, the convergence up to the boundary is impossible in general. In fact we would usually anticipate a thin transition layer near the boundary, called boundary layer, where the viscous flows change value from its mainstream value to the boundary value. (See for instance Temam and Wang (1996) for an illustration, and Schlichting (1979) for more information.) A useful observation is that only adjustments normal to the boundary is needed, or only the normal derivatives need to be large, not necessarily the tangential derivatives. Due to the presence of boundary layer, uniform estimates in derivatives are not available. Nor it is possible to derive uniform in space estimates since there is no-known maximum principle type technique available due to the presence of the pressure term which makes the problem a global one. Another approach is along the line of Prandtl: one assume that the viscous and inviscid solutions are close to each other in the interior of the domain away from the boundary (layer). Within the boundary layer, the viscosity is important, and the motion is approximated by the so-called Prandtl's equation which is a heuristic approximation of the Navier-Stokes equations under the assumption that the flow scales as $\mathbf{u}^{\nu}=\mathbf{g}(x, y, z / \sqrt{\nu}, t)$ where we assumed that $z=0$ is the boundary (see Prandtl 1905). In order to justify this picture, one has to prove the well-posedness of the Prandtl system (local in time at least), and then use matched asymptotic expansion or some other techniques to establish the validity of the Prandtl expansion and the inviscid limit. Unfortunately the well-posedness of the Prandtl equation is very difficult (see for instance Oleinik (1963), E and Engquist (1997) among other). Even in the case of well-posed Prandtl equation, there is no known successful matching for non-trivial flows. (See for instance E (2000) for a survey.) A slightly different approach is the so-called corrector approach along the lines of Vishik and Lyusternik (1957) and Lions (1973). The idea here is to write the viscous solution $\mathbf{u}^{\nu}$ as the sum of the inviscid solution $\mathbf{u}^{0}$ 
and a corrector $\theta$. Inserting this into the viscous equation, assuming that the corrector $\theta$ is concentrated in the boundary layer and satisfying certain scaling property, we are able to derive a Prandtl type equation for the corrector, called corrector equation. One then proceed to prove the well-posedness of the corrector (Prandtl type) equation and the closeness of the viscous solution and $\mathbf{u}^{0}+\theta$. This is closely related to multi-scale (in this case two scale) expansion. Usually this is also a difficult task. The advantage here is that no matching is needed. Indeed in the case of uniformly non-characteristic boundary case this procedure was carried out successfully by Temam and Wang (1999). The main ingredient of success there is that the Prandtl type (corrector) equation is a linear elliptic system and the boundary layer is of thickness linearly proportional to the kinematic viscosity $\nu$. However in the characteristic boundary case as is considered here, even the well-posedness of the Prandtl equation is beyond our reach now except for some special cases. See for instance Oleinik and Samokhin (1999) and the references therein.

In an article published in 1984, T. Kato proved a very interesting result regarding the validity of inviscid limit, i.e., the convergence of the solutions of the Navier-Stokes equations to that of the Euler equations at vanishing kinematic viscosity (for the special case of $\mathbf{b} \equiv 0$, thus no Taylor-Couette type flows allowed):

Kato 1984: a necessary and sufficient condition for the validity of the inviscid limit is the vanishing of the following energy dissipation rate in a viscous sublayer of thickness $c \nu$, i.e.,

$$
\nu \int_{0}^{T} \int_{\Gamma_{c \nu}}\left|\nabla \mathbf{u}^{\nu}(\vec{x}, t)\right|^{2} d \vec{x} d t \rightarrow 0, \text { as } \nu \rightarrow 0,
$$

where $\Gamma_{c \nu}$ is the $c \nu$ neighborhood of the wall $\Gamma=\partial \Omega$, and $\mathrm{c}$ is an arbitrary constant of order one with the unit $[1 / U]$. Kato's result indicates that the problem of inviscid limit depends on the behavior of the viscous solution in the viscous sublayer only where the viscosity is supposed to dominate. This suggest that the Prandtl equation may not be central to the question of inviscid limit. Roughly speaking, after been translated into the language of correctors, Kato found an ansatz for the corrector. His ansatz has nothing to do with the Prandtl type equations since the scalings are totally different. Kato's ansatz was later applied to problem of Ekman layer by Grenier and Masmoudi (1997) among others. Unfortunately Kato's result is not easily applicable since it is hard to estimate the energy dissipation rate. The pur- 
pose of this note is to present a Kato type result with the total gradient replaced by the tangential derivatives only. The difference between the result here and the result of Kato as well as our previous note (Temam and Wang 1998) are the following: 1. we use tangential derivatives only (with a slightly thicker boundary layer), a small quantity in classical boundary layer theory, while Kato needs the whole gradient; 2. we treat more general and physically more interesting boundary condition here including Taylor Couette type flow while both Kato (1984) and our previous note treat stationary solid wall $(\mathbf{u}=0$, on $\Gamma)$ only; 3 . we have a necessary and sufficient condition here (with a slightly thicker boundary layer though) while only a sufficient condition is presented in our previous note (Temam and Wang 1998).

This is inspired by classical laminar boundary layer theory which asserts that the tangential (to the boundary) variation of the velocity is much smaller than the normal (to the boundary) variation of the velocity, (see for instance Schlichting (1979),) the rigorous bound on energy dissipation rate (see for instance Doering and Gibbon (1995) among others) and the laboratory experiment on shear driven flows (see Lathrop, Fineberg and Swinney (1992)). It also agrees with recent carefully designed numerical experiments by $\mathrm{H}$. Johnson, J-G Liu and W. E (2000).

\section{The Main Result and Applications}

In this section we state our main result and its application to three examples.

Theorem 1 Suppose that we have a smooth solution $\mathbf{u}^{0}$ of the Euler system (6) on the time interval $[0, T]$. Let $\mathbf{u}^{\nu}$ be a Leray-Hopf solution to the NavierStokes system (1). Then the following are equivalent

$$
\mathbf{u}^{\nu} \rightarrow \mathbf{u}^{0}, \text { in } C\left([0, T], L^{2}(\Omega)\right)
$$

- There exists $\delta(\nu)$ such that

$$
\begin{aligned}
\lim _{\nu \rightarrow 0} \frac{\nu}{\delta(\nu)} & =0 \\
\lim _{\nu \rightarrow 0} \nu \int_{0}^{T} \int_{\Gamma_{\delta}}\left|\nabla_{\tau} \mathbf{u}_{\tau}^{\nu}\right|^{2} & =0
\end{aligned}
$$


- There exists $\delta(\nu)$ such that

$$
\begin{aligned}
\lim _{\nu \rightarrow 0} \frac{\nu}{\delta(\nu)} & =0 \\
\lim _{\nu \rightarrow 0} \nu \int_{0}^{T} \int_{\Gamma_{\delta}}\left|\nabla_{\tau} u_{n}^{\nu}\right|^{2} & =0
\end{aligned}
$$

where $\nabla_{\tau}$ denotes tangential (to the boundary) derivatives and $\mathbf{u}_{\tau}^{\nu}$ denotes the tangential component(s) of the velocity while $u_{n}^{\nu}$ denotes the normal component of the velocity, using natural curvilinear coordinates in the boundary layer.

Remark One of the choice of $\delta(\nu)$ is $\frac{h}{R e} \log R e$ where $h$ is the characteristic length and $R e=\frac{h U}{\nu}$ is the Reynolds number. This is the well-known thickness of viscous sublayer predicted by von Karman (1930) logarithmic profile. Notice that the layer here is thicker than the one used by Kato $(h / R e)$.

Remark The short time existence of smooth solutions of the Euler system (6) can be found in Temam (1975) provided that the data are smooth.

As immediate application of our result we have

Example 1 (Radial symmetric disk flows, see for instance Matsui (1994)) In this case $\Omega$ is a disk of radius $R$ and the velocity is radial symmetric, i.e.,

$$
\mathbf{u}^{\nu}=\mathbf{u}^{\nu}(r, t)
$$

Notice that the tangential derivative is the derivative in the $\vec{e}_{\phi}$ direction, i.e.,

$$
\nabla_{\tau}=\frac{\partial}{\partial \phi}
$$

Hence the second and third conditions in the theorem are satisfied which implies the convergence. This case is not the most interesting one since the nonlinear term drops out under this radial symmetry. The velocity equation and the pressure are decoupled.

Example 2 (Plane parallel channel flow) Here we consider a special type of channel flow. Let $\Omega$ be a channel bounded between $z=0$ and $z=h$. Suppose that the third component of the velocity field is identically zero, i.e.,

$$
\mathbf{u}^{\nu}=\left(u_{1}^{\nu}, u_{2}^{\nu}, 0\right)
$$


Thus the flows are parallel to the plane $z=0$. Again the condition for convergence is satisfied in the theorem above and hence the convergence must be true. Unlike the previous example, this case includes some nonlinear situations. For instance for the special choice of

$$
\mathbf{u}^{\nu}=\left(u_{1}^{\nu}(z, t), u_{2}^{\nu}(x, z, t), 0\right)
$$

the equations reduce to a weakly coupled nonlinear system

$$
\begin{aligned}
\frac{\partial u_{1}^{\nu}}{\partial t}-\nu \frac{\partial^{2} u_{1}^{\nu}}{\partial z^{2}} & =f_{1}(z, t), \\
\frac{\partial u_{2}^{\nu}}{\partial t}+u_{1}^{\nu} \frac{\partial u_{2}^{\nu}}{\partial x}-\nu \frac{\partial^{2} u_{1}^{\nu}}{\partial z^{2}}-\nu \frac{\partial^{2} u_{1}^{\nu}}{\partial x^{2}} & =f_{2}(x, z, t) .
\end{aligned}
$$

Example 3 (Parallel Pipe flow) An example similar to example 2 is a parallel pipe flow. Here we consider a pipe with circular cross-section with $x$-axis being the axis of the pipe. We assume that the flow is parallel to the axis of the pipe all the time. More specifically we consider the special case of

$$
\mathbf{u}^{\nu}=u_{\phi}^{\nu}(x, r, t) \mathbf{e}_{\phi}+u_{x}^{\nu}(x, r, t) \mathbf{e}_{x}
$$

in the cylindrical coordinates with $\phi$ being the angle and $r$ being the distance to the axis of the pipe. Since the normal flow (in the direction of $\mathbf{e}_{r}$ ) is identically zero, the convergence is true. Again in a special subcase the system reduce to a weakly coupled nonlinear system just as in example 2 .

\section{Corollary and Related Results}

In this section we present several consequences and related results of our main result. One of the interesting consequences indicates the numerical difficulty in verifying the inviscid limit. Basically, one has to resolve a small scale of the order $h / R e$ in the direction parallel to the wall in the numerical experiment in order to be able to say anything about the inviscid limit. Here $h$ is the characteristic length of the domain (in the channel case it is the width of the channel) and $R e$ is the Reynolds number. Notice Kato's result implies that one has to resolve small scale of the order $h / R e$ in the direction normal to the wall only. Another interesting result indicates that if the 
pressure variance along the wall does not grow too fast (less than $R e^{3 / 2}$ ) in the two dimensional case, then the convergence must be true. This indicates that even in the case with adverse pressure gradient (thus boundary layer separates and the Prandtl theory cease to valid), the convergence may still be true. This is a further indication that the problem of inviscid limit in the presence of boundary may not be related to the validity of the Prandtl theory. (Though we believe (yet to be proved) that the Prandtl theory should be valid for a short period of time.)

For simplicity we will consider channel flow (flat boundary) with periodicity in the horizontal $(x)$ direction.

Corollary 1 Suppose we have a numerical scheme preserving the property that the time averaged energy dissipation rate is bounded above independent of the kinematic viscosity. Let $\mathbf{u}^{k}$ be the solutions of a sequence of numerical experiments with kinematic viscosity $\nu_{k} \rightarrow 0$ and horizontal cut-off wave number $K_{k} \rightarrow \infty$. The experiment is horizontally under-resolved in the sense that

$$
\lim _{k \rightarrow \infty} \nu_{k} K_{k}=0
$$

then at least one of the assumptions in our main result is satisfied for the discrete solutions.

Proof Let

$$
\mathbf{u}^{k} \sim \sum_{j=0}^{K_{k}} \mathbf{u}^{k, j}(z, t) e^{2 \pi i j x / L}
$$

be the numerical solutions where $L$ is the period in the horizontal direction. Set

$$
\delta_{k}=\frac{\nu_{k}}{U}\left(\frac{L U}{\nu_{k} K_{k}}\right)^{\frac{1}{2}}
$$

we have

$$
\frac{\nu_{k}}{\delta_{k}} \rightarrow 0
$$

We have, thanks to Poincaré inequality,

$$
\begin{aligned}
\nu_{k} \int_{0}^{T} \int_{\Gamma_{\delta_{k}}}\left|D_{x} u_{2}^{k}\right|^{2} & \leq \delta_{k}^{2} \nu_{k} \int_{0}^{T} \int_{\Gamma_{\delta_{k}}}\left|D_{x} D_{z} u_{2}^{k}\right|^{2} \\
& =\delta_{k}^{2} \nu_{k} \int_{0}^{T} \int_{\Gamma_{\delta_{k}}}\left|D_{x}^{2} u_{1}^{k}\right|^{2}
\end{aligned}
$$




$$
\begin{aligned}
& \leq \delta_{k}^{2} K_{k}^{2} \frac{4 \pi^{2}}{L^{2}} \nu_{k} \int_{0}^{T} \int_{\Gamma_{\delta_{k}}}\left|D_{x} u_{1}^{k}\right|^{2} \\
& \leq \kappa \nu_{k} K_{k} \\
& \rightarrow 0
\end{aligned}
$$

where we have used the assumption on time averaged energy dissipation rate. The fact that the time averaged energy dissipation rate is bounded above by a constant independent of the kinematic viscosity $\nu$ for the continuous equation can be found in Doering and Constantin (1992). This leads to our result which proves the corollary.

Remark: A more rigorous result would require a proof of the convergence of the discrete solutions to that of the Euler equations under the horizontally under-resolved assumption (16). The proof for this result as well as some other related results will appear elsewhere.

Our second corollary indicates that even if there is adverse pressure gradient, it is still possible for the Euler equation to be the inviscid limit of the Navier-Stokes equations. This indicates that even if the Prandtl theory cease to valid, the inviscid limit could still be true. This is another indication that the problem of inviscid limit may not be related to the validity of Prandtl theory.

Corollary $\mathbf{2}$ We consider the two dimensional case with $\mathbf{b} \equiv 0$. Suppose that the pressure of the viscous flows satisfies one of the following conditions

$$
\begin{aligned}
\lim _{\nu \rightarrow 0} \nu^{\frac{3}{2}} \int_{0}^{T} \int_{\Gamma}\left|D_{\tau} p^{\nu}\right|^{2} & =0, \\
\lim _{\nu \rightarrow 0} \nu \int_{0}^{T}\left|p^{\nu}\right|_{H^{\frac{1}{2}}(\Gamma)}^{2} & =0,
\end{aligned}
$$

then $\mathbf{u}^{\nu}$ converges to $\mathbf{u}^{0}$ in $C\left([0, T], L^{2}\right)$.

Proof: For the two dimensional case with $\mathbf{b} \equiv 0$, we have

$$
\int_{\Omega}(\mathbf{u} \cdot \nabla) \mathbf{u} \cdot \Delta \mathbf{u}=0
$$

for any smooth $\left(H^{2}\right)$ divergence free function. (see for instance Constantin and Foias, 1988.) (This identity may not hold for the case with nontrivial $\mathbf{b}$ or in the three dimensional case.) 
For simplicity we will consider flat boundary case at $z=0$.

We multiply the Navier-Stokes equations (1) by $-\Delta \mathbf{u}^{\nu}$ and integrate over $\Omega$. The nonlinear term vanishes by the identity cited above.

For the right hand side we have

$$
\begin{aligned}
-\int_{\Omega} \mathbf{f} \cdot \Delta \mathbf{u}^{\nu} & \leq|\mathbf{f}|_{L^{2}}\left|\Delta \mathbf{u}^{\nu}\right|_{L^{2}} \\
& \leq \frac{\nu}{4}\left|\Delta \mathbf{u}^{\nu}\right|_{L^{2}}^{2}+\frac{1}{\nu}|\mathbf{f}|_{L^{2}}^{2} .
\end{aligned}
$$

For the pressure term we have either

$$
\begin{aligned}
\int_{\Omega} \nabla p^{\nu} \cdot \Delta \mathbf{u}^{\nu} & =\int_{\Gamma} p^{\nu} \Delta \mathbf{u}^{\nu} \cdot \mathbf{n} \\
& \leq\left|p^{\nu}\right|_{H^{\frac{1}{2}}(\Gamma)}\left|\Delta \mathbf{u}^{\nu} \cdot \mathbf{n}\right|_{H^{-\frac{1}{2}(\Gamma)}} \\
& \leq\left|p^{\nu}\right|_{H^{\frac{1}{2}(\Gamma)}}\left|\Delta \mathbf{u}^{\nu}\right|_{L^{2}} \\
& \leq \frac{\nu}{4}\left|\Delta \mathbf{u}^{\nu}\right|_{L^{2}}^{2}+\frac{1}{\nu}\left|p^{\nu}\right|_{H^{\frac{1}{2}(\Gamma)}}^{2}
\end{aligned}
$$

or

$$
\begin{aligned}
\int_{\Omega} \nabla p^{\nu} \cdot \Delta \mathbf{u}^{\nu} & =\int_{\Gamma} p^{\nu} \Delta \mathbf{u}^{\nu} \cdot \mathbf{n} \\
& =-\int_{\Gamma} p^{\nu} \Delta u_{2}^{\nu} \\
& =-\int_{\Gamma} p^{\nu} \frac{\partial^{2} u_{2}^{\nu}}{\partial z^{2}} \\
& =\int_{\Gamma} p^{\nu} \frac{\partial^{2} u_{1}^{\nu}}{\partial x \partial z} \\
& =-\int_{\Gamma} \frac{\partial p^{\nu}}{\partial x} \frac{\partial u_{1}^{\nu}}{\partial z} \\
& \leq\left|\frac{\partial p^{\nu}}{\partial x}\right|_{L^{2}(\Gamma)}\left|\frac{\partial u_{1}^{\nu}}{\partial z}\right|_{L^{2}(\Gamma)} \\
& \leq\left|\frac{\partial p^{\nu}}{\partial x}\right|_{L^{2}(\Gamma)}\left|\frac{\partial u_{1}^{\nu}}{\partial z}\right|_{L^{2}}^{\frac{1}{2}}\left|\frac{\partial u_{1}^{\nu}}{\partial z}\right|_{H^{1}}^{\frac{1}{2}} \\
& \leq \frac{\nu}{4}\left|\Delta \mathbf{u}^{\nu}\right|_{L^{2}}^{2}+\left|u_{1}^{\nu}\right|_{H^{1}}^{2}+\frac{1}{\nu^{\frac{1}{2}}}\left|\frac{\partial p^{\nu}}{\partial x}\right|_{L^{2}(\Gamma)}^{2} .
\end{aligned}
$$

These implies, together with the assumptions,

$$
\frac{d}{d t}\left|\nabla \mathbf{u}^{\nu}\right|_{L^{2}}^{2}+\nu\left|\Delta \mathbf{u}^{\nu}\right|_{L^{2}}^{2} \leq \kappa\left|\nabla \mathbf{u}^{\nu}\right|_{L^{2}}^{2}+\mathbf{o}\left(\nu^{-2}\right),
$$


and hence we have

$$
\lim _{\nu \rightarrow 0} \nu^{3} \int_{0}^{T}\left|\Delta \mathbf{u}^{\nu}\right|_{L^{2}}^{2}=0
$$

or equivalently

$$
\eta(\nu) \equiv \frac{\nu^{3}}{L U^{5} T} \int_{0}^{T}\left|\Delta \mathbf{u}^{\nu}\right|_{L^{2}}^{2} \rightarrow 0, \text { as } \nu \rightarrow 0 .
$$

Setting

$$
\alpha(\nu)=\eta(\nu)^{\frac{1}{3}},
$$

we have

$$
\frac{\nu}{\delta}=\alpha U \rightarrow 0, \text { as } \nu \rightarrow 0
$$

Moreover we have

$$
\begin{aligned}
\nu \int_{0}^{T} \int_{\Gamma_{\delta}}\left|\frac{\partial u_{1}^{\nu}}{\partial x}\right|^{2} & \leq \nu \delta^{2} \int_{0}^{T} \int_{\Gamma_{\delta}}\left|\frac{\partial^{2} u_{1}^{\nu}}{\partial x \partial z}\right|^{2} \\
& \leq \frac{\nu^{3}}{\alpha^{2} U^{2}} \int_{0}^{T}\left|\Delta \mathbf{u}^{\nu}\right|_{L^{2}}^{2} \\
& =L U^{3} T \eta(\nu)^{\frac{1}{3}} \\
& \rightarrow 0, \text { as } \nu \rightarrow 0 .
\end{aligned}
$$

Hence the assumptions in the main theorem are satisfied and the corollary follows.

\section{Sketch of the Proof}

Our proof is along the line of Kato (1984) and Temam and Wang (1998) with some modification. The basic idea is to construct a so called Background ground flow with a free parameter $\alpha$ which interpolates between the viscous sublayer (Kato type result) and laminar boundary layer (Prandtl theory).

For simplicity we will consider channel flow (flat boundary) and two dimensional case only. The case with curved boundary can be treated in the same way as in our previous work Temam and Wang (1998) using curvilinear coordinates. The three dimensional case is very similar to our work on energy dissipation rate Wang $(1997,2000)$.

Our approach is close to the idea of Vishik and Lyusternik (1957) (see also Lions 1973) in the sense that we seek a corrector which approximates 
the difference between the viscous and inviscid solution. Hence it is slightly different from Kato's (1984) approach.

Throughout this section, $\kappa$ will denote a generic constant independent of the kinematic viscosity $\nu$.

We prove the necessity first. For this purpose let $\phi$ be a fixed incompressible flow that matches $\mathbf{b}$ on the boundary of the domain. The existence of such flows is classical (see for instance the textbook by Temam 1984). Now consider

$$
\begin{aligned}
\mathbf{v}^{\nu} & =\mathbf{u}^{\nu}-\phi, \\
\mathbf{v}^{0} & =\mathbf{u}^{0}-\phi .
\end{aligned}
$$

Notice that $\mathbf{v}^{\nu}$ satisfies the following equation

$$
\begin{aligned}
\frac{\partial \mathbf{v}^{\nu}}{\partial t}+\left(\mathbf{v}^{\nu} \cdot \nabla\right) \mathbf{v}^{\nu}+\left(\mathbf{v}^{\nu} \cdot \nabla\right) \phi+(\phi \cdot \nabla) \mathbf{v}^{\nu}-\nu \Delta \mathbf{v}^{\nu} & =\mathbf{f}-\frac{\partial \phi}{\partial t}-(\phi \cdot \nabla) \phi+\psi(22) \\
\operatorname{div} \mathbf{v}^{\nu} & =0, \\
\mathbf{v}^{\nu} & =0 \text { on } \Gamma, \\
\mathbf{v}^{\nu} & =\mathbf{u}_{0}-\phi \text { at } t=0 .
\end{aligned}
$$

The usual energy inequality on Leray-Hopf type weak solution implies that

$$
\frac{1}{2} \frac{d}{d t}\left|\mathbf{v}^{\nu}\right|_{L^{2}}^{2}+\int_{\Omega}\left(\mathbf{v}^{\nu} \cdot \nabla\right) \phi \cdot \mathbf{v}^{\nu}+\nu\left|\nabla \mathbf{v}^{\nu}\right|_{L^{2}}^{2} \leq \int_{\Omega}\left(\mathbf{f}-\frac{\partial \phi}{\partial t}-(\phi \cdot \nabla) \phi+\nu \Delta \phi\right) \cdot \mathbf{v}^{\nu} .
$$

Integrating in time we deduce

$$
\frac{1}{2}\left|\mathbf{v}^{\nu}(T)\right|_{L^{2}}^{2}+\int_{0}^{T} \int_{\Omega}\left(\mathbf{v}^{\nu} \cdot \nabla\right) \phi \cdot \mathbf{v}^{\nu}+\nu \int_{0}^{T} \int_{\Omega}\left|\nabla \mathbf{v}^{\nu}\right|^{2} \leq \int_{0}^{T} \int_{\Omega}\left(\mathbf{f}-\frac{\partial \phi}{\partial t}-(\phi \cdot \nabla) \phi+\nu \Delta \phi\right) \cdot \mathbf{v}^{\nu}+\frac{1}{2}\left|\mathbf{u}_{0}-\phi\right|_{L^{2}}^{2}
$$

On the other hand the inviscid solution (after translation by $\phi) \mathbf{v}^{0}$ satisfies the following equality (as long as it remains smooth enough)

$\frac{1}{2}\left|\mathbf{v}^{0}(T)\right|_{L^{2}}^{2}+\int_{0}^{T} \int_{\Omega}\left(\mathbf{v}^{0} \cdot \nabla\right) \phi \cdot \mathbf{v}^{0}=\int_{0}^{T} \int_{\Omega}\left(\mathbf{f}-\frac{\partial \phi}{\partial t}-(\phi \cdot \nabla) \phi+\nu \Delta \phi\right) \cdot \mathbf{v}^{0}+\frac{1}{2}\left|\mathbf{u}_{0}-\phi\right|_{L^{2}}^{2}$

Since $\mathbf{u}^{\nu} \rightarrow \mathbf{u}^{0}$ in $C\left([0, T], L^{2}\right)$ we deduce that

$$
\mathbf{v}^{\nu} \rightarrow \mathbf{v}^{0} \text { in } C\left([0, T], L^{2}\right),
$$


which further implies that

$$
\limsup _{\nu \rightarrow 0} \nu \int_{0}^{T} \int_{\Omega}\left|\nabla \mathbf{v}^{\nu}\right|^{2}=0 .
$$

This implies the necessarily condition in our main theorem.

Next we move on to the sufficiency of our condition. For simplicity we will consider two dimensional domain with flat boundary only. Without loss of generality we assume $z=0$ is the boundary and the domain lies in the upper half plane.

We introduce a corrector with a free parameter $\alpha$ which allows us to interpolate between the laminar boundary layer theory of Prandtl and the viscous sublayer. The family of stream function is defined as

$$
\psi^{\nu}(x, z, t)=\left(b_{1}(x, 0, t)-u_{1}^{0}(x, 0, t)\right) \int_{0}^{z} \rho\left(\frac{\alpha U s}{\nu}\right) d s,
$$

with a corresponding part for the upper boundary $(z=h$ for the channel case) and with the typical velocity $U$ defined as

$$
U=\max _{[0, T] \times \Gamma}\left|b_{1}-u_{1}^{0}\right| .
$$

The corresponding velocity field (corrector) is defined as

$$
\theta^{\nu}(x, z, t)=\operatorname{curl} \psi^{\nu}(x, z, t)=\left(\frac{\partial \psi^{\nu}}{\partial z},-\frac{\partial \psi^{\nu}}{\partial x}\right)
$$

plus a corresponding part at $z=h$, where $\rho$ is a cut-off type function having the property

$$
\begin{array}{r}
\rho \in C^{\infty}[0, \infty), \\
\rho(0)=1, \\
\rho^{\prime}(0)=0, \\
\operatorname{supp} \rho \subset[0,1), \\
\int_{0}^{1} \rho=0, \\
|\rho|_{L^{\infty}} \leq 1, \\
\left|\rho^{\prime}\right|_{L^{\infty}} \leq 2 .
\end{array}
$$


Notice that our choice of corrector (background flow) has a back flow.

Notice that by our explicit construction, the stream function (and hence the velocity field) is supported in the $\frac{\nu}{\alpha U}$ neighborhood of the boundary. Hence it is plausible to call $\delta=\frac{\nu}{\alpha U}$ the thickness of the boundary layer associated with the background flow.

Our next step is to consider the adjusted difference

$$
\mathbf{w}^{\nu}=\mathbf{u}^{\nu}-\mathbf{u}^{0}-\theta^{\nu} .
$$

We intend to prove that $\mathbf{w}^{\nu}$ approaches zero at vanishing viscosity provided the assumptions in our theorem are satisfied.

The adjusted difference $\mathbf{w}^{\nu}$ satisfies the following equation

$$
\begin{aligned}
\frac{\partial \mathbf{w}^{\nu}}{\partial t}+\left(\mathbf{u}^{\nu} \cdot \nabla\right) \mathbf{w}^{\nu}-\nu \Delta \mathbf{w}^{\nu}+\nabla q^{\nu}= & -\frac{\partial \theta^{\nu}}{\partial t}-\left(\theta^{\nu} \cdot \nabla\right) \theta^{\nu}-\left(\mathbf{w}^{\nu} \cdot \nabla\right) \theta^{\nu}-\left(\mathbf{w}^{\nu} \cdot \nabla\right) \mathbf{u}^{0} \\
& -\left(\theta^{\nu} \cdot \nabla\right) \mathbf{u}^{0}+\nu \Delta \mathbf{u}^{0}+\nu \Delta \theta^{\nu}, \\
\operatorname{div} \mathbf{w}^{\nu}= & 0, \\
\mathbf{w}^{\nu}= & 0, \text { on } \Gamma, \\
\mathbf{w}^{\nu}= & 0, \text { at } t=0 .
\end{aligned}
$$

Thanks to the explicit construction of our corrector $\theta^{\nu}$ we have

$$
\begin{aligned}
\left|\frac{\partial \theta^{\nu}}{\partial t}\right|_{L^{2}}^{2} & \leq U_{t}^{2} \frac{L \nu}{\alpha U}+U_{t x}^{2} \frac{L \nu^{3}}{\alpha^{3} U^{3}}, \\
\left|\left(\theta^{\nu} \cdot \nabla\right) \theta^{\nu}\right|_{L^{2}}^{2} & \leq 5 U^{2} U_{x}^{2} \frac{L \nu}{\alpha U}+U^{2} U_{x x}^{2} \frac{L \nu^{3}}{\alpha^{3} U^{3}}+U_{x}^{4} \frac{L \nu^{3}}{\alpha^{3} U^{3}}, \\
\left|\left(\theta^{\nu} \cdot \nabla\right) \mathbf{u}^{0}\right|_{L^{2}}^{2} & \leq\left|\nabla u^{0}\right|_{L^{\infty}}^{2}\left(U^{2} \frac{L \nu}{\alpha U}+U_{x}^{2} \frac{L \nu^{3}}{\alpha^{3} U^{3}}\right), \\
\left|\nabla \theta^{\nu}\right|_{L^{2}}^{2} & \leq 2 U_{x}^{2} \frac{L \nu}{\alpha U}+U^{2} \frac{L \alpha U}{\nu}+U_{x x}^{2} \frac{L \nu^{3}}{\alpha^{3} U^{3}},
\end{aligned}
$$

where

$$
\begin{aligned}
U_{t} & =\max _{[0, T] \times \Gamma}\left|\frac{\partial b_{1}}{\partial t}-\frac{\partial u_{1}^{0}}{\partial t}\right|, \\
U_{x} & =\max _{[0, T] \times \Gamma}\left|\frac{\partial b_{1}}{\partial x}-\frac{\partial u_{1}^{0}}{\partial x}\right| \\
U_{t x} & =\max _{[0, T] \times \Gamma}\left|\frac{\partial^{2} b_{1}}{\partial t \partial x}-\frac{\partial^{2} u_{1}^{0}}{\partial t \partial x}\right|,
\end{aligned}
$$




$$
U_{x x}=\max _{[0, T] \times \Gamma}\left|\frac{\partial^{2} b_{1}}{\partial x^{2}}-\frac{\partial^{2} u_{1}^{0}}{\partial x^{2}}\right| .
$$

This implies, together with the standard multiplier method,

$$
\begin{aligned}
\frac{1}{2} \frac{d}{d t}\left|\mathbf{w}^{\nu}\right|_{L^{2}}^{2}+\nu\left|\nabla \mathbf{w}^{\nu}\right|_{L^{2}}^{2} \leq & \nu \sqrt{2 U_{x}^{2} \frac{L \nu}{\alpha U}+U^{2} \frac{L \alpha U}{\nu}+U_{x x}^{2} \frac{L \nu^{3}}{\alpha^{3} U^{3}}}\left|\nabla \mathbf{w}^{\nu}\right|_{L^{2}} \\
& +U_{t}^{2} \frac{L \nu}{\alpha U}+U_{t x}^{2} \frac{L \nu^{2}}{\alpha^{2} U^{2}} \\
& 5 U^{2} U_{x}^{2} \frac{L \nu}{\alpha U}+U^{2} U_{x x}^{2} \frac{L \nu^{3}}{\alpha^{3} U^{3}}+U_{x}^{4} \frac{L \nu^{3}}{\alpha^{3} U^{3}} \\
& \left|\nabla \mathbf{u}^{0}\right|_{L^{\infty}}^{2}\left(U^{2} \frac{L \nu}{\alpha U}+U_{x}^{2} \frac{L \nu^{3}}{\alpha^{3} U^{3}}\right) \\
& +\nu^{2}\left|\Delta \mathbf{u}^{0}\right|_{L^{2}}^{2} \\
& +\left(1+\left|\nabla \mathbf{u}^{0}\right|_{L^{\infty}}\right)\left|\mathbf{w}^{\nu}\right|_{L^{2}}^{2} \\
& +\int_{\Omega}\left(\mathbf{w}^{\nu} \cdot \nabla\right) \mathbf{w}^{\nu} \cdot \theta^{\nu}
\end{aligned}
$$

Notice the nonlinear term can be rewritten as

$\int_{\Omega}\left(\mathbf{w}^{\nu} \cdot \nabla\right) \mathbf{w}^{\nu} \cdot \theta^{\nu}=\int_{\Omega} w_{1}^{\nu} \frac{\partial w_{1}^{\nu}}{\partial x} \theta_{1}^{\nu}+\int_{\Omega} w_{3}^{\nu} \frac{\partial w_{1}^{\nu}}{\partial z} \theta_{1}^{\nu}+\int_{\Omega} w_{1}^{\nu} \frac{\partial w_{3}^{\nu}}{\partial x} \theta_{3}^{\nu}+\int_{\Omega} w_{3}^{\nu} \frac{\partial w_{3}^{\nu}}{\partial z} \theta_{3}^{\nu}$,

and hence we have the following estimates on the nonlinear term, thanks to the explicit construction of the corrector,

$$
\begin{aligned}
& 2 \int_{\Omega} w_{1}^{\nu} \frac{\partial w_{1}^{\nu}}{\partial x} \theta_{1}^{\nu}=\int_{\Omega} \frac{\partial}{\partial x}\left(w_{1}^{\nu}\right)^{2} \theta_{1}^{\nu} \\
& =-\int_{\Omega}\left(w_{1}^{\nu}\right)^{2} \frac{\partial \theta_{1}^{\nu}}{\partial x} \\
& \leq U_{x}\left|w_{1}^{\nu}\right|_{L^{2}}^{2} \\
& 2 \int_{\Omega} w_{3}^{\nu} \frac{\partial w_{1}^{\nu}}{\partial z} \theta_{1}^{\nu} \leq 2 U\left|w_{3}^{\nu}\right|_{L^{2}\left(\Gamma_{\delta}\right)}\left|\frac{\partial w_{1}^{\nu}}{\partial z}\right|_{L^{2}\left(\Gamma_{\delta}\right)} \\
& \leq 2 U \delta\left|\frac{\partial w_{3}^{\nu}}{\partial z}\right|_{L^{2}\left(\Gamma_{\delta}\right)}\left|\frac{\partial w_{1}^{\nu}}{\partial z}\right|_{L^{2}\left(\Gamma_{\delta}\right)} \\
& =\frac{2 \nu}{\alpha}\left|\frac{\partial w_{1}^{\nu}}{\partial x}\right|_{L^{2}\left(\Gamma_{\delta}\right)}\left|\frac{\partial w_{1}^{\nu}}{\partial z}\right|_{L^{2}\left(\Gamma_{\delta}\right)} \\
& \leq \frac{\nu}{4}\left|\frac{\partial w_{1}^{\nu}}{\partial z}\right|_{L^{2}\left(\Gamma_{\delta}\right)}^{2}+\frac{4 \nu}{\alpha^{2}}\left|\frac{\partial w_{1}^{\nu}}{\partial x}\right|_{L^{2}\left(\Gamma_{\delta}\right)}^{2},
\end{aligned}
$$




$$
\begin{aligned}
2 \int_{\Omega} w_{1}^{\nu} \frac{\partial w_{3}^{\nu}}{\partial x} \theta_{3}^{\nu} & \leq \kappa \frac{\nu}{\alpha}\left|w_{1}^{\nu}\right|_{L^{2}\left(\Gamma_{\delta}\right)}\left|\frac{\partial w_{3}^{\nu}}{\partial x}\right|_{L^{2}\left(\Gamma_{\delta}\right)} \\
& \leq \frac{\nu}{4}\left|\frac{\partial w_{3}^{\nu}}{\partial x}\right|_{L^{2}\left(\Gamma_{\delta}\right)}^{2}+\kappa \frac{\nu}{\alpha^{2}}\left|w_{1}^{\nu}\right|_{L^{2}\left(\Gamma_{\delta}\right)}^{2}
\end{aligned}
$$

Similarly

$$
2 \int_{\Omega} w_{3}^{\nu} \frac{\partial w_{3}^{\nu}}{\partial z} \theta_{3}^{\nu} \leq \frac{\nu}{4}\left|\frac{\partial w_{3}^{\nu}}{\partial z}\right|_{L^{2}\left(\Gamma_{\delta}\right)}^{2}+\kappa \frac{\nu}{\alpha^{2}}\left|w_{3}^{\nu}\right|_{L^{2}\left(\Gamma_{\delta}\right)}^{2} .
$$

This implies that

$$
\frac{d}{d t}\left|\mathbf{w}^{\nu}\right|_{L^{2}}^{2} \leq \mathbf{o}(1)+\kappa\left|\mathbf{w}^{\nu}\right|_{L^{2}}^{2}+\alpha L U^{3}+\frac{8 \nu}{\alpha^{2}}\left|\frac{\partial w_{1}^{\nu}}{\partial x}\right|_{L^{2}\left(\Gamma_{\delta}\right)}^{2},
$$

which further implies, thanks to the Gronwall inequality,

$$
\left\|\mathbf{w}^{\nu}\right\|_{L^{\infty}\left(0, T ; L^{2}(\Omega)\right)} \leq \mathbf{o}(1)+\kappa\left(\alpha L U^{3}+\frac{8 \nu}{\alpha^{2}} \frac{1}{T} \int_{0}^{T} \int_{\Gamma_{\delta}}\left|\frac{\partial w_{1}^{\nu}}{\partial x}\right|^{2}\right)^{\frac{1}{2}},
$$

provided that

$$
\frac{\nu}{\alpha^{2}} \rightarrow 0, \text { as } \nu \rightarrow 0
$$

or

$$
\frac{\delta^{2}}{\nu} \rightarrow 0, \text { as } \nu \rightarrow 0
$$

If this is not satisfied, (the boundary layer for the background flow is too thick), just replace it with a background flow with narrower support.

It is easy to see that the expression

$$
\alpha L U^{3}+\frac{8}{\alpha^{2}} \frac{\nu}{T} \int_{0}^{T} \int_{\Gamma_{\delta}}\left|\frac{\partial w_{1}^{\nu}}{\partial x}\right|^{2}
$$

as a function of $\alpha$ attains its minimum when

$$
\alpha=\alpha_{c t}=\left(\frac{16}{L U^{3}} \frac{\nu}{T} \int_{0}^{T} \int_{\Gamma_{\delta}}\left|\frac{\partial w_{1}^{\nu}}{\partial x}\right|^{2}\right)^{\frac{1}{3}}
$$

provided that the value of $\frac{\nu}{T} \int_{0}^{T} \int_{\Gamma_{\delta}}\left|\frac{\partial w_{1}^{\nu}}{\partial x}\right|^{2}$ is fixed.

In the case of

$$
\alpha_{c t} \geq \alpha
$$


we have

$$
\delta_{c t}=\frac{\nu}{\alpha_{c t} U} \leq \frac{\nu}{\alpha U}=\delta
$$

and hence

$$
\begin{aligned}
\alpha_{c t} L U^{3}+\frac{8}{\alpha_{c t}^{2}} \frac{\nu}{T} \int_{0}^{T} \int_{\Gamma_{\delta_{c t}}}\left|\frac{\partial w_{1}^{\nu}}{\partial x}\right|^{2} & \leq \alpha_{c t} L U^{3}+\frac{8}{\alpha_{c t}^{2}} \frac{\nu}{T} \int_{0}^{T} \int_{\Gamma_{\delta}}\left|\frac{\partial w_{1}^{\nu}}{\partial x}\right|^{2} \\
& =L U^{3}\left(\frac{18 \nu}{L U^{3} T} \int_{0}^{T} \int_{\Gamma_{\delta}}\left|\frac{\partial w_{1}^{\nu}}{\partial x}\right|^{2}\right)^{\frac{1}{3}} .
\end{aligned}
$$

On the other hand, if

$$
\alpha_{c t}<\alpha
$$

we have

$$
\left(\frac{16}{L U^{3}} \frac{\nu}{T} \int_{0}^{T} \int_{\Gamma_{\delta}}\left|\frac{\partial w_{1}^{\nu}}{\partial x}\right|^{2}\right)^{\frac{1}{3}}<\alpha
$$

or

$$
\frac{\nu}{T} \int_{0}^{T} \int_{\Gamma_{\delta}}\left|\frac{\partial w_{1}^{\nu}}{\partial x}\right|^{2}<\frac{\alpha^{3} L U^{3}}{16},
$$

which further implies

$$
\alpha L U^{3}+\frac{8}{\alpha^{2}} \frac{\nu}{T} \int_{0}^{T} \int_{\Gamma_{\delta}}\left|\frac{\partial w_{1}^{\nu}}{\partial x}\right|^{2} \leq \frac{3}{2} \alpha L U^{3} .
$$

Combining the two cases and (41) we deduce that

$$
\left\|\mathbf{w}^{\nu}\right\|_{L^{\infty}\left(0, T ; L^{2}(\Omega)\right)} \leq \mathbf{o}(1)+\kappa L U^{3} \max \left\{\alpha,\left(\frac{\nu}{L U^{3} T} \int_{0}^{T} \int_{\Gamma_{\delta}}\left|\frac{\partial w_{1}^{\nu}}{\partial x}\right|^{2}\right)^{\frac{1}{3}}\right\} .
$$

This completes the proof of the part of the main theorem on tangential derivative of tangential velocity.

For the part with assumption on the tangential derivative of the normal velocity, we introduce an auxiliary function

$$
\tilde{\theta}(z)=\int_{z}^{\delta}\left(\theta_{1}(\tau)\right)^{2} d \tau=\frac{\nu}{\alpha U}\left(b_{1}(x, 0, t)-u_{1}^{0}(x, 0, t)\right)^{2} \int_{\frac{\alpha z U}{\nu}}^{1} \rho^{2}(s) d s .
$$

We then have the following alternative estimates on the nonlinear terms

$$
2 \int_{\Omega} w_{3}^{\nu} \frac{\partial w_{1}^{\nu}}{\partial z} \theta_{1}^{\nu} \leq 2\left|\frac{\partial w_{1}^{\nu}}{\partial z}\right|_{L^{2}\left(\Gamma_{\delta}\right)}\left|w_{3}^{\nu} \theta_{1}^{\nu}\right|_{L^{2}\left(\Gamma_{\delta}\right)},
$$


and

$$
\begin{aligned}
\int_{\Omega}\left(w_{3}^{\nu} \theta_{1}^{\nu}\right)^{2} & =\int_{\Gamma_{\delta}}\left(w_{3}^{\nu}\right)^{2} \frac{\partial}{\partial z} \tilde{\theta}(z) \\
& =-\int_{\Gamma_{\delta}} 2 w_{3}^{\nu} \frac{\partial w_{3}^{\nu}}{\partial z} \tilde{\theta}(z) \\
& =\int_{\Gamma_{\delta}} 2 w_{3}^{\nu} \frac{\partial w_{1}^{\nu}}{\partial x} \tilde{\theta}(z) \\
& =-2 \int_{\Omega_{\delta}}\left(w_{1}^{\nu} \frac{\partial w_{3}^{\nu}}{\partial x} \tilde{\theta}(z)+w_{1}^{\nu} w_{3}^{\nu} \frac{\partial \tilde{\theta}(z)}{\partial x}\right) \\
& \leq 2\left(\left|\frac{w_{1}^{\nu}}{z}\right|_{L^{2}\left(\Gamma_{\delta}\right)}\left|\frac{\partial w_{3}^{\nu}}{\partial x}\right|_{L^{2}\left(\Gamma_{\delta}\right)}|z \tilde{\theta}(z)|_{L^{\infty}\left(\Gamma_{\delta}\right)}+\left|\frac{w_{1}^{\nu}}{z}\right|_{L^{2}\left(\Gamma_{\delta}\right)}\left|\frac{w_{3}^{\nu}}{z}\right|_{L^{2}\left(\Gamma_{\delta}\right)}\left|z^{2} \frac{\partial \tilde{\theta}(z)}{\partial x}\right|_{L^{\infty}\left(\Gamma_{\delta}\right)}\right) \\
& \leq \kappa\left(\left|\frac{\partial w_{1}^{\nu}}{\partial z}\right|_{L^{2}\left(\Gamma_{\delta}\right)}\left|\frac{\partial w_{3}^{\nu}}{\partial x}\right|_{L^{2}\left(\Gamma_{\delta}\right)} \frac{\nu^{2}}{\alpha^{2}}+\left|\frac{\partial w_{1}^{\nu}}{\partial z}\right|_{L^{2}\left(\Gamma_{\delta}\right)}\left|\frac{\partial w_{3}^{\nu}}{\partial z}\right|_{L^{2}\left(\Gamma_{\delta}\right)} \frac{\nu^{3}}{\alpha^{3}}\right) .
\end{aligned}
$$

Hence

$$
\begin{aligned}
2 \int_{\Omega} w_{3}^{\nu} \frac{\partial w_{1}^{\nu}}{\partial z} \theta_{1}^{\nu} & \leq \kappa \frac{\nu}{\alpha}\left|\frac{\partial w_{1}^{\nu}}{\partial z}\right|_{L^{2}}^{\frac{3}{2}}\left|\frac{\partial w_{3}^{\nu}}{\partial x}\right|_{L^{2}\left(\Gamma_{\delta}\right)}^{\frac{1}{2}}+\kappa\left(\frac{\nu}{\alpha}\right)^{\frac{3}{2}}\left|\nabla \mathbf{w}^{\nu}\right|_{L^{2}}^{2} \\
& \leq \frac{\nu}{4}\left|\nabla \mathbf{w}^{\nu}\right|_{L^{2}}^{2}+\kappa \frac{\nu}{\alpha^{4}}\left|\frac{\partial w_{3}^{\nu}}{\partial x}\right|_{L^{2}\left(\Gamma_{\delta}\right)}^{2},
\end{aligned}
$$

provided

$$
\lim _{\nu \rightarrow 0} \frac{\nu}{\alpha^{3}}=0,
$$

or equivalently,

$$
\lim _{\nu \rightarrow 0} \frac{\delta^{3}}{\nu^{2}}=0 .
$$

Again this is a restriction on the thickness of the boundary layer for the corrector. If this is violated, just replace it with a corrector with thinner boundary layer.

The rest of the proof is parallel to the previous case.

\section{Acknowledgments}

This work was supported in part by a Faculty Development Fund from Iowa State University and the National Science Foundation. Part of the work 
was finished while the author was a visiting member of The Institute of Mathematical Sciences, The Chinese University of Hong Kong in the summer of 2000. The support from the Institute is greatly appreciated. The author would like to thank Weinan E for several very helpful comments.

\section{References}

1. P. Constantin and C. Foias, 1988, Navier-Stokes Equations, Chicago University Press.

2. Ch. R. Doering and P. Constantin, 1992, Energy dissipation in sheardriven turbulence, Phys. Rev. Lett. 69, 1648-1651.

3. Ch. Doering and J.D. Gibbons, 1995, Applied Analysis of the NavierStokes Equations, Cambridge University Press.

4. W. E, 2000, Boundary layer theory and the zero-viscosity limit of the Navier-Stokes equations, Acta Math Sinica, vol. 16, No. 2, pp. 207218.

5. W. E and B. Engquist, 1997, Blow-up of solutions of the unsteady Prandtl's equation, Comm. Pure Appl. Math. 50, no. 12, 1287-1293.

6. E. Grenier and N. Masmoudi, 1997, Ekman layers of rotating fluids, the case of well prepared initial data, Comm. Partial Differential Equations 22, no. 5-6, pp. 953-975.

7. H. Johnston, J.-G. Liu and W. E, 2000 A Numerical Study of the ZeroViscosity Limit of the Flow Around a Cylinder, in preparation.

8. Th. von Kármán, 1930, Mechanische Ähnlichkeit und Turbulenz, in Collected Works of Theodore von Kármán, vol. 2, 337-346, London, Butterworths Scientific Publications, 1956.

9. T. Kato, 1984, Remarks on the zero viscosity limit for nonstationary Navier-Stokes flows with boundary, in Seminar on PDE, edited by S.S. Chern, Springer, NY. 
10. O.A. Ladyzhenskaya, 1969, The Mathematical Theory of Viscous Incompressible Flows,2ed Gordon and Breach, New York.

11. D. Lathrop, J. Fineberg and H. Swinney, 1992, Transition to shear driven turbulence in Couette-Taylor flow, Phys. Rev. A 46, 6390-6405.

12. J.L. Lions, 1973, Perturbations singulières dans les problèmes aux limites et en controle optimal, Lecture Notes in Math., vol 323, SpringerVerlag, New York.

13. N. Masmoudi, 1998, The Euler limit of the Navier-Stokes equations, and rotating fluids with boundary, Arch. Rational Mech. Anal. 142, no. 4, 375-394.

14. S. Matsui, 1994, Example of zero viscosity limit for two dimensional nonstationary Navier-Stokes flows with boundary, Japan J. Industrial and Appl. Math., 11, pp. 155 - 173.

15. O. Oleinik, 1963, The Prandtl system of equations in boundary layer theory, Soviet Math. Dokl., 4, pp. 583 - 586.

16. O. Oleinik and V. Samokhin, 1999, Mathematical models in boundary layer theory, Chapman and Hall/CRC, Boca Raton, FL.

17. L. Prandtl, 1905, Verber Flüssigkeiten bei sehr kleiner Reibung, Verk. III Intem. Math. Kongr. Heidelberg, 484-491, Teuber, Leibzig.

18. M. Sammartino and R. E. Calflisch, 1995, 1996, Zero viscosity limit for analytic solutions of the Navier-Stokes equations, Proceedings of the VIII International Conference on Waves and Stability in Continuous Media, Part II (Palermo, 1995). Rend. Circ. Mat. Palermo (2) Suppl. No. 45, part II (1996), 595-605.

19. H. Schlichting, 1979, Boundary Layer Theory, Yed, McGraw-Hill, New York.

20. R. Temam, 1975, On the Euler equations of incompressible perfect fluids, J. Funct. Anal., 20, pp. 32-43.

21. R. Temam, 1984, Navier-Stokes Equations, North-Holland, 3rd Edition, Amsterdam. 
22. R. Temam and X. Wang, 1996, Asymptotic Analysis of Oseen Type Equations in a Channel at Small Viscosity, Indiana University Mathematics Journal, 45, pp. 863-916.

23. R. Temam and X. Wang, 1998, On the behavior of the solutions of the Navier-Stokes equations at vanishing viscosity, Annali della Scuola Normale Superiore de Pisa, volume dedicated to the memory of E. De Giorgi, Serie IV, Vol. XXV (1998), 807-828.

24. R. Temam and X. Wang, 1999, Boundary layers associated with the Navier-Stokes equations: the non-characteristic boundary case, The Institute for Scientific Computing and Applied Math Preprint 9908.

25. M. Vishik and Lyusternik, 1957, Regular degeneration and boundary layer for linear differential equations with small parameter, Uspekki Mat. Nauk, vol 12, pp. 3-122, 1957.

26. X. Wang, 2000, Effect of tangential derivatives in the boundary layer on time averaged energy dissipation rate, Physica D 144(2000) 142-153. 